\title{
Longitudinal dispersion in a horizontal subsurface flow constructed wetland: a numerical solution
}
A. Araújo ${ }^{1}$
E. Sousa ${ }^{2}$
A. Albuquerque ${ }^{3}$

(Received 24 July 2008; revised 4 November 2008)

\begin{abstract}
We present a numerical solution for the dead zone model which describes the solute transport in a subsurface and horizontal flow constructed wetland. This model is a system of two mass balance equations for two conceptual areas: the main channel and the storage zone. We use finite difference schemes to determine the numerical solution of the system and we study its convergence by presenting properties related to the stability and accuracy of the schemes. Concerning the experimental results, the magnitude of the longitudinal dispersion and the extension of dead volumes is estimated for clean conditions and after a certain operating period under organic loading conditions. The results showed a considerable amount of longitudinal dispersion
\end{abstract}

http://anziamj . austms.org.au/ojs/index.php/ANZIAMJ/article/view/1367 gives this article, (c) Austral. Mathematical Soc. 2008. Published November 18, 2008. ISSN 1446-8735. (Print two pages per sheet of paper.) 
through the bed, which was very strong near the feeding point, indicating the occurrence of mixing and significant presence of dead zones and short-circuiting. This approach is expected to be useful to determine operating conditions, such as, the control of the incoming organic loading, and also to avoid the increase of dead zones as a means to improve treatment performance.

\section{Contents}

1 Introduction

C340

2 Model problem

C342

3 Finite differences discretization

C343

4 Convergence of the finite differences

C344

4.1 Consistency . . . . . . . . . . . . . . . . . . . C C344

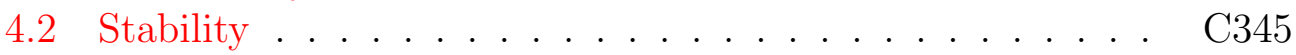

5 Materials and methods

C347

6 Numerical and experimental results

C349

References

C351

\section{Introduction}

Constructed wetlands are considered a technical, economical and environmental sustainable solution for wastewater treatment in small communities since they are efficient with pollutant removal and have high filter capacity $[4,5]$. The most used solution presented a subsurface and horizontal flow 
bed (SSHF) and it is recognized to have a good ecological integration. However, with packing media used as a bed it may become frequently clogged due to factors whose interrelations are not well known. The transport of solutes in the bed is, therefore, difficult to study since there are many factors which affect the transport mechanisms, such as the development of roots, media characteristics (for example, type of material, porosity and physical properties such as sorption), excessive biofilm growth, hydraulic and organic loadings, chemical and biochemical transformations and accumulation of solids.

A complex interaction of these processes in the porous media may stimulate the development of immobile areas, dead volumes, hydraulic shortcircuiting, internals recirculation and changes in dispersion conditions. These affect the solute distribution through media and, therefore, the treatment efficiency may be adversely affected. The analysis of flow patterns for different bed characteristics (for example, with and without vegetation) and organic loadings is essential for a better understanding of the transport of solutes in such systems and, therefore, to allow accurate prediction of treatment. A reliable mean of evaluating the extension of those mechanisms is to carry out tracer tests through the bed and analyse the exit concentration curves with time (breakthrough curve) through numerical solutions of transport equations. The hydrodynamic characteristics of the bed, in terms of flow regime, extension of dead areas and longitudinal dispersion may be analysed by estimating parameters such as the Peclet number $(\mathrm{Pe})$ or the ratio of dead zones volumes $(\epsilon)$. Therefore, the objective of this paper is to propose a numerical solution for the dead zone model to simulate the solute transport in a SSHF constructed wetland.

The dead zone model is a system of two mass balance equations for two conceptual areas: the main channel and the storage zone. The main channel is defined as that portion of the stream in which advection and dispersion are the dominant transport mechanisms. The storage zone is defined as the portion of the stream that contributes to transient storage. Within the main channel, solutes are transported downstream by advection and dispersion. 
Advection and dispersion are not included in the storage zones, where downstream transport is negligible.

\section{Model problem}

For a conservative solute the equations governing longitudinal transport and mixing in a stream are $[2,6,7$, e.g.]

$$
\begin{aligned}
& \frac{\partial C}{\partial t}+u \frac{\partial C}{\partial x}=D \frac{\partial^{2} C}{\partial x^{2}}+\epsilon T^{-1}\left(C_{s}-C\right), \\
& \frac{\partial C_{s}}{\partial t}=T^{-1}\left(C-C_{s}\right),
\end{aligned}
$$

with $\mathrm{C}$ and $\mathrm{C}_{\mathrm{s}}$ as solute concentration $(\mathrm{mg} / \mathrm{l})$ in main stream and storage zones, $\boldsymbol{u}$ the mean effective flow velocity $(\mathrm{m} / \mathrm{h})$, where $\mathrm{h}$ denotes hour, $\mathrm{D}$ the dispersion coefficient $\left(\mathrm{m}^{2} / \mathrm{h}\right), \epsilon$ the dimensionless ratio of dead zone volume and main volume per unit length and $\mathrm{T}$ is an exchange parameter $(\mathrm{h})$ related to mean dead zone residence time. The later two parameters are

$$
\epsilon=\frac{A_{s}}{A} \quad \text { and } \quad T^{-1}=\alpha \frac{A}{A_{s}},
$$

with $A$ denoting the flow cross-section $\left(\mathrm{m}^{2}\right), A_{s}$ the storage zone crosssectional area $\left(\mathrm{m}^{2}\right)$ and $\alpha$ the storage zone exchange coefficient $\left(\mathrm{h}^{-1}\right)$.

For our particular problem the initial conditions are

$$
C(x, 0)=0 \text { and } C_{s}(x, 0)=0 \text { for } x>0 .
$$

Although in most of the experimental procedures there are some uncertainty about what happens at the upstream boundary, that is, near the tracer injection point, we evaluate it as a function

$$
\mathrm{C}(0, \mathrm{t})=\mathrm{g}(\mathrm{t}), \quad \mathrm{t}>0 .
$$


The boundary condition, $g(t)$, represents the solute concentration at the inflow boundary and is the following exponential decay

$$
g(t)=C_{0} e^{-Q t / V_{\text {inj }}},
$$

where $V_{\text {inj }}$ denotes the volume of injected tracer, $C_{0}$ is the concentrated solution injected at the beginning and $\mathrm{Q}$ denotes the flow rate $\left(\mathrm{m}^{3} / \mathrm{h}\right)$. This condition is obtained considering that the inflow concentration is governed by the differential equation, which describes the inflow decay by a rate of $Q / V_{i n j}$,

$$
\frac{\mathrm{dg}}{\mathrm{dt}}=-\frac{\mathrm{Q}}{\mathrm{V}_{\mathrm{inj}}} \mathrm{g}, \text { with } \mathrm{g}(0)=\mathrm{C}_{0} .
$$

\section{Finite differences discretization}

To derive a finite difference scheme we suppose there are approximations $C^{n}:=\left\{C_{i}^{n}\right\}$ to the values $C\left(x_{i}, t_{n}\right)$ and $C_{s}^{n}:=\left\{C_{s, i}^{n}\right\}$ to the values $C_{s}\left(x_{i}, t_{n}\right)$ at the mesh points $x_{i}=i \Delta x, i=0,1,2, \ldots, N$ and $t_{n}=n \Delta t, n=$ $0,1,2, \ldots, M$. Let us also consider the central and second difference operators, $\Delta_{0} C_{i}^{n}:=\frac{1}{2}\left(C_{i+1}^{n}-C_{i-1}^{n}\right)$ and $\delta^{2} C_{i}^{n}:=C_{i+1}^{n}-2 C_{i}^{n}+C_{i-1}^{n}$.

Using second order centered finite differences operators in space and the trapezoidal rule in time (Crank-Nicolson scheme), we obtain

$$
\begin{aligned}
\frac{C_{i}^{n+1}-C_{i}^{n}}{\Delta t}= & \frac{1}{2}\left(-\frac{u}{\Delta x} \Delta_{0} C_{i}^{n+1}+\frac{D}{\Delta x^{2}} \delta^{2} C_{i}^{n+1}+\epsilon T^{-1}\left(C_{s, i}^{n+1}-C_{i}^{n+1}\right)\right) \\
& +\frac{1}{2}\left(-\frac{u}{\Delta x} \Delta_{0} C_{i}^{n}+\frac{D}{\Delta x^{2}} \delta^{2} C_{i}^{n}+\epsilon T^{-1}\left(C_{s, i}^{n}-C_{i}^{n}\right)\right) \\
\frac{C_{s, i}^{n+1}-C_{s, i}^{n}}{\Delta t}= & \frac{1}{2} T^{-1}\left(C_{i}^{n+1}-C_{s, i}^{n+1}\right)+\frac{1}{2} T^{-1}\left(C_{i}^{n}-C_{s, i}^{n}\right) .
\end{aligned}
$$

In order to avoid implicitness we use the decoupling procedure of the two equations suggested by Runkel and Chapra [7]. First we rearrange the terms 
in (7) in the form

$$
C_{s, i}^{n+1}=(1-2 \gamma) C_{s, i}^{n}+\gamma\left(C_{i}^{n}+C_{i}^{n+1}\right),
$$

where $\gamma=\Delta t /(2 T+\Delta t)$. Secondly, replacing (8) in (6), we obtain

$$
\begin{aligned}
C_{i}^{n+1}= & C_{i}^{n}+\frac{1}{2}\left(-v \Delta_{0}+\mu \delta^{2}-\Delta t \in T^{-1}(1-\gamma)\right) C_{i}^{n+1} \\
& +\frac{1}{2}\left(-v \Delta_{0}+\mu \delta^{2}-\Delta t \epsilon T^{-1}(1-\gamma)\right) C_{i}^{n}+\Delta t \in T^{-1}(1-\gamma) C_{s, i}^{n},(9)
\end{aligned}
$$

for $v=V \Delta t / \Delta x$ (Courant number) and $\mu=D \Delta t / \Delta x^{2}$ (Fourier number). Since $\Delta t \epsilon T^{-1}(1-\gamma)=2 \epsilon \gamma$, we write the system (8)-(9) in the form

$$
\begin{aligned}
\mathcal{A} C_{i}^{n+1} & =\mathcal{B} C_{i}^{n}+2 \epsilon \gamma C_{s, i}^{n}, \\
C_{s, i}^{n+1} & =(1-2 \gamma) C_{s, i}^{n}+\gamma\left(C_{i}^{n}+C_{i}^{n+1}\right),
\end{aligned}
$$

where $\mathcal{A}$ and $\mathcal{B}$ are the difference operators $\mathcal{A}=1-\frac{1}{2}\left(-v \Delta_{0}+\mu \delta^{2}-2 \epsilon \gamma\right)$, and $\mathcal{B}=1+\frac{1}{2}\left(-\frac{\gamma}{2} \Delta_{0}+\mu \delta^{2}-2 \epsilon \gamma\right)$.

\section{Convergence of the finite differences}

In order to study the convergence of the finite differences schemes (6)-(7), we consider the approach by Verwer and Sanz-Serna [8]. In this approach to prove the convergence we need to establish the consistency of the finite difference schemes as well as to prove the stability of the spatial and full discretizations.

\subsection{Consistency}

Let $v=v(x, t)$ and $v_{s}=v_{s}(x, t)$ be the solutions to our system of equations. We denote by $\tau_{i}^{n}$ and $\tau_{s, i}^{n}$ the truncation errors associated with the 
equations (1) and (2) respectively. We have

$$
\begin{aligned}
& \tau_{i}^{n}=\frac{v_{i}^{n+1}-v_{i}^{n}}{\Delta t}-\frac{1}{2}\left(-\frac{u}{\Delta x} \Delta_{0} v_{i}^{n+1}+\frac{D}{\Delta x^{2}} \delta^{2} v_{i}^{n+1}\right) \\
& -\frac{1}{2}\left(-\frac{u}{\Delta x} \Delta_{0} v_{i}^{n}+\frac{D}{\Delta x^{2}} \delta^{2} v_{i}^{n}\right)-\frac{1}{2} \epsilon T^{-1}\left(v_{s, i}^{n+1}+v_{s, i}^{n}\right)-\frac{1}{2} \epsilon T^{-1}\left(v_{i}^{n+1}+v_{i}^{n}\right) \\
& \tau_{s, i}^{n}=\frac{v_{s, i}^{n+1}-v_{s, i}^{n}}{\Delta t}-\frac{1}{2} T^{-1}\left(v_{s, i}^{n+1}+v_{s, i}^{n}\right)-\frac{1}{2} T^{-1}\left(v_{i}^{n+1}+v_{i}^{n}\right)
\end{aligned}
$$

Therefore

$$
\begin{aligned}
\tau_{i}^{n}= & \left(\frac{\partial v}{\partial t}\right)_{i}^{n+1 / 2}+\mathcal{O}\left(\Delta t^{2}\right)+u\left(\frac{\partial v}{\partial x}\right)_{i}^{n+1 / 2}-D\left(\frac{\partial^{2} v}{\partial x^{2}}\right)_{i}^{n+1 / 2}+\mathcal{O}\left(\Delta x^{2}\right) \\
& +\mathcal{O}\left(\Delta t^{2}\right)-\epsilon T^{-1}\left(v_{s}-v\right)_{i}^{n+1 / 2}+\mathcal{O}\left(\Delta x^{2}\right) \\
\tau_{s, i}^{n}= & \left(\frac{\partial v}{\partial t}\right)_{i}^{n+1 / 2}+\mathcal{O}\left(\Delta t^{2}\right)-T^{-1}\left(v-v_{s}\right)_{i}^{n+1 / 2}+\mathcal{O}\left(\Delta t^{2}\right) .
\end{aligned}
$$

The scheme's consistency is of order $\mathcal{O}\left(\Delta t^{2}\right)+\mathcal{O}\left(\Delta x^{2}\right)$.

\subsection{Stability}

We consider the problem (1)-(2) written in the form

$$
\frac{\partial \mathbf{C}}{\partial t}+\mathbf{U} \frac{\partial \mathbf{C}}{\partial x}=\mathbf{D} \frac{\partial^{2} \mathbf{C}}{\partial x^{2}}+\mathbf{E C},
$$

where $\mathbf{C}$ represents the exact solution $\mathbf{C}(x, t)=\left(C(x, t), C_{s}(x, t)\right)$ and

$$
\mathbf{U}=\left[\begin{array}{ll}
u & 0 \\
0 & 0
\end{array}\right], \quad \mathbf{D}=\left[\begin{array}{ll}
\mathrm{D} & 0 \\
0 & 0
\end{array}\right] \quad \text { and } \quad \mathbf{E}=\mathrm{T}^{-1}\left[\begin{array}{cc}
-\epsilon & \epsilon \\
1 & -1
\end{array}\right] \text {. }
$$

The stability of the spatial discretization is based on the existence of a bounded logarithmic matrix norm [3]. The stability of the full discretization 
is proved using the concept of C-stability, which is linked with stability in the Lax-Richtmyer sense [3].

Let us first consider the spatial discretization. The semi-discrete system of ordinary differential equations (12) is written in the form

$$
\frac{\mathrm{dC}}{\mathrm{dt}}=\mathrm{MC},
$$

where $\mathbf{C}$ now represents the solution at the discrete points, that is, $\mathbf{C}\left(x_{i}, t\right)=$ $\left(C\left(x_{i}, t\right), C_{s}\left(x_{i}, t\right)\right)$. The matrix

$$
\mathbf{M}=\frac{1}{2 \Delta x} \mathrm{D}_{\mathrm{c}} \otimes \mathbf{U}+\frac{1}{\Delta \mathrm{x}^{2}} \mathrm{D}_{2} \otimes \mathbf{D}+\mathrm{I} \otimes \mathbf{E},
$$

where $\otimes$ denotes the Kronecker product. Since this discretization is of second order and therefore consistent with our problem, to prove the convergence of the spatial discretization, according to Dekker and Verwer [3], we just need to prove the existence of a constant $\mu_{\max }$ which is independent of the grid spacing and such that $\mu_{\infty}[\mathbf{M}] \leq \mu_{\max }$. For $A=\left[a_{i j}\right]$, the logarithmic matrix norm

$$
\mu_{\infty}[\mathbf{A}]=\max _{i}\left(a_{i i}+\sum_{j \neq i}\left|a_{i j}\right|\right) .
$$

According to the structure of the matrix $\mathbf{M}$, we easily conclude that

$$
\mu_{\infty}[\mathbf{M}]=\max \left\{\left|\frac{\mathrm{u}}{2 \Delta \mathrm{x}}+\frac{\mathrm{D}}{\Delta \mathrm{x}^{2}}\right|-\frac{2 \mathrm{D}}{\Delta \mathrm{x}^{2}}+\left|-\frac{\mathrm{u}}{2 \Delta \mathrm{x}}+\frac{\mathrm{D}}{\Delta \mathrm{x}^{2}}\right|\right\} .
$$

Note that, if $u \Delta x / D>2$, then $\mu_{\infty}[\mathbf{M}]=\mu_{\max }=0$. Otherwise, $\mu_{\infty}[\mathbf{M}] \leq$ $\mu_{\max }=u^{2} \mathrm{D}$. Then $\mu_{\infty}[\mathbf{M}]$ is bounded and the spatial discretization is convergent.

For the full discretization we consider the Crank-Nicolson scheme

$$
\mathbf{C}^{\mathrm{n}+1}=\mathbf{C}^{\mathrm{n}}+\frac{\Delta \mathrm{t}}{2} \mathbf{M}\left(\mathbf{C}^{\mathrm{n}+1}+\mathbf{C}^{\mathrm{n}}\right) .
$$


In order to establish the C-stability of this scheme we prove the existence of a positive real number $\Delta t_{0}=\Delta t_{0}(\Delta x)$ and a real constant $C_{0}$, independent of $\Delta \mathrm{t}$ and $\Delta \mathrm{x}$, such that for each $\left.\Delta \mathrm{t} \in] 0, \Delta \mathrm{t}_{0}\right]$ and each approximated solution $\mathbf{C}$ and $\tilde{\mathbf{C}}$ we have $\left\|\tilde{\mathbf{C}}^{n+1}-\mathbf{C}^{n+1}\right\|_{\infty} \leq\left(1+\mathrm{C}_{0} \Delta t\right)\left\|\tilde{\mathbf{C}}^{n}-\mathbf{C}^{\mathfrak{n}}\right\|_{\infty}$.

Let $\mathbf{C}^{n}$ and $\mathbf{C}^{n+1}$, and $\tilde{\mathbf{C}}^{n}$ and $\tilde{\mathbf{C}}^{n+1}$ denote two solutions of the CrankNicolson scheme, that is, they verify (14). Then

$$
\left(\mathbf{I}-\frac{\Delta \mathrm{t}}{2} \mathbf{M}\right)\left(\tilde{\mathbf{C}}^{\mathrm{n}+1}-\mathbf{C}^{\mathrm{n}+1}\right)=\left(\mathbf{I}-\frac{\Delta \mathrm{t}}{2} \mathbf{M}\right)\left(\tilde{\mathbf{C}}^{\mathrm{n}}-\mathbf{C}^{\mathfrak{n}}\right) .
$$

By applying the infinity norm, $\|\cdot\|_{\infty}$, for $\Delta t \mu_{\max }<2$, we obtain

$$
\left\|\tilde{\mathbf{C}}^{\mathfrak{n}+1}-\mathbf{C}^{\mathfrak{n}+1}\right\|_{\infty} \leq\left(\frac{2+\Delta \mathrm{t} \mu_{\max }}{2-\Delta \mathrm{t} \mu_{\max }}\right)\left\|\tilde{\mathbf{C}}^{\mathfrak{n}}-\mathbf{C}^{\mathfrak{n}}\right\|_{\infty} .
$$

Therefore, according to the results above we conclude the following.

Theorem 1 For $\frac{\mathrm{u} \Delta \mathrm{x}}{\mathrm{D}}>2$, the method (6)-(7) is unconditionally convergent. For $\frac{\mathrm{u} \Delta \mathrm{x}}{\mathrm{D}} \leq 2$, if $\Delta \mathrm{t} \leq \frac{2}{\mathrm{u}^{2} \mathrm{D}}$, then the method is convergent.

Note that, $u \Delta x / D$ is the mesh Peclet number. When the mesh Peclet number is less or equal to 2 , the upper bound to the time step is a nonrestrictive condition, since $\boldsymbol{u}$ and $\mathrm{D}$ are less than one.

\section{Materials and methods}

A laboratory SSHF system with $2.0 \mathrm{~m} \times 0.80 \mathrm{~m} \times 0.50 \mathrm{~m}$ was used to carry out two series of tracer tests at the mean flow velocity of $0.0047 \mathrm{~m} \mathrm{~h}^{-1}$. The submerged media was composed of gravel $(0.20 \mathrm{~m}$ in depth and porosity of 0.4$)$. Series I was executed with the bed without vegetation and Series II with the 


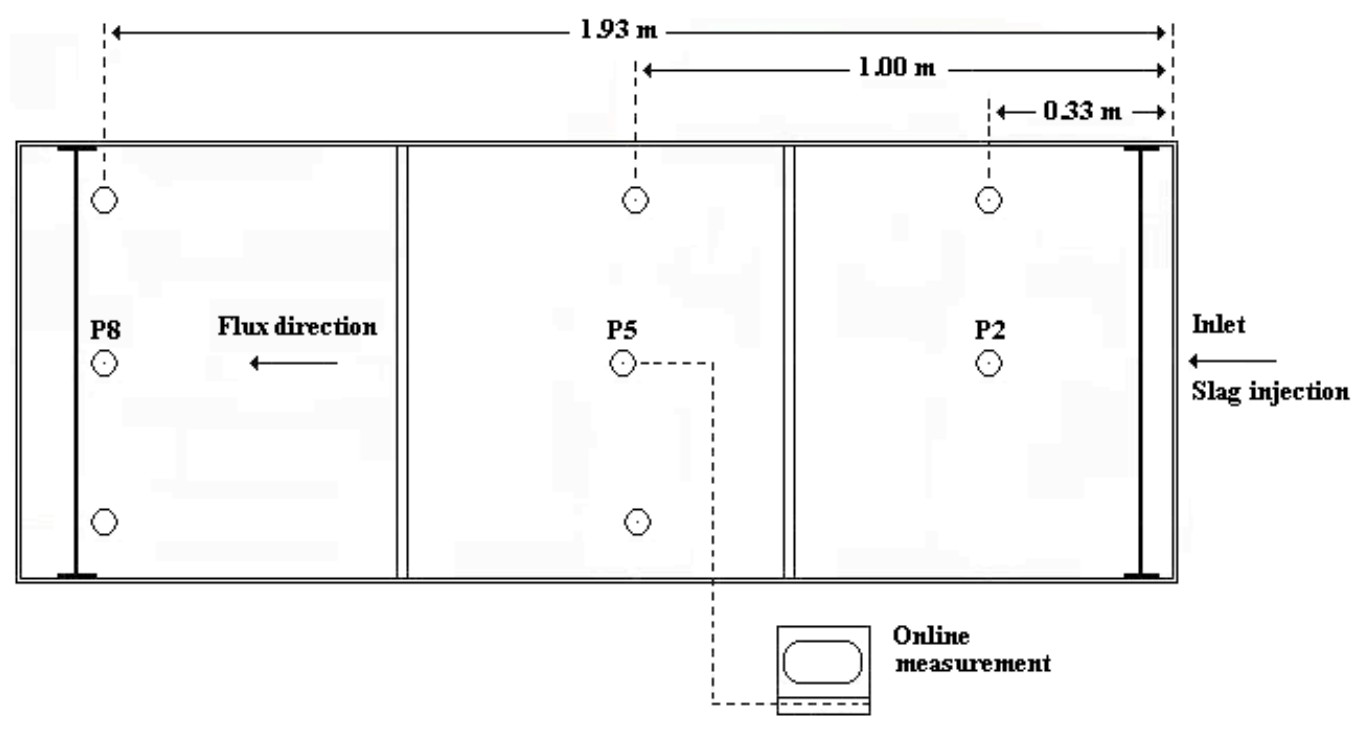

FIGURE 1: Schematic representation of the laboratory device (plant).

bed already colonized with Phragmites australis after an operation period of eight months at organic loads ranging from 0.42 to $1.38 \mathrm{~g} \mathrm{COD} \mathrm{m}^{-2} \mathrm{~h}^{-1}$ (roots were well developed and spread over the bed).

Each Series included three assays with tap water and a quickly injection of $0.5 \mathrm{l}$ of sodium chloride with a concentration of $100 \mathrm{gl}^{-1}\left(\mathrm{C}_{0}\right)$ for three bed lengths: Inlet-P2 $(0.33 \mathrm{~m})$, Inlet-P5 $(1.00 \mathrm{~m})$ and Inlet-P8 $(1.93 \mathrm{~m})$. The response was evaluated by online measurement of a conductivity TetraCon 325 probe and Multi 340i WTW meter (Figure 1). The time duration of each assay was up to 15 times the theoretical hydraulic retention time until no significant conductivity were observed at the measuring point. 


\begin{tabular}{|c|cc|lllll|}
\hline Assay & L & tfinal & $\epsilon$ & $\mathrm{T}$ & $\mathrm{D}$ & $\mathrm{Pe}$ & $\mathrm{DaI}$ \\
\hline I.P2 & 0.33 & 100 & 15 & 1000 & $3.0 \times 10^{-4}$ & 5.6 & 1.04 \\
I.P5 & 1.00 & 250 & 26 & 3650 & $3.0 \times 10^{-4}$ & 29.5 & 0.84 \\
I.P8 & 1.93 & 500 & 25 & 6000 & $4.1 \times 10^{-4}$ & 41 & 0.96 \\
\hline II.P2 & 0.33 & 100 & 15 & 1300 & $1.4 \times 10^{-4}$ & 12 & 0.80 \\
II.P5 & 1.00 & 250 & 26 & 5700 & $1.5 \times 10^{-4}$ & 50 & 0.63 \\
II.P8 & 1.93 & 500 & 25 & 7000 & $2.8 \times 10^{-4}$ & 60 & 0.83 \\
\hline
\end{tabular}

TABle 1: Results for Series I and II.

\section{$6 \quad$ Numerical and experimental results}

This section presents the experimental results and the numerical solution which simulates the transport mechanism that occurs experimentally. These numerical results were obtained by adjusting the parameters $\epsilon, \mathrm{T}, \mathrm{D}$ and $\mathrm{Pe}$ to the experimental results, according to Table 1.

Let DaI be the experimental Damköhler number

$$
\mathrm{DaI}=\frac{\alpha\left(1+\mathrm{A} / \mathrm{A}_{s}\right) \mathrm{L}}{\mathrm{u}}=\frac{\mathrm{T}^{-1} \epsilon\left(1+\epsilon^{-1}\right) \mathrm{L}^{2}}{\mathrm{PeD}}=\frac{(1+\epsilon) \mathrm{L}^{2}}{\mathrm{TPeD}},
$$

where $L$ is the flow path length and $P e=u L / D$ is the Peclet number.

Wagner and Harvey [9] showed that the experimental Damköhler number, DaI, is a valuable indicator of the reliability of storage zone crosssectional area and exchange coefficient estimates. The use of the experimental Damköhler number was adapted from similar subsurface transport research by Bahr and Rubin [1]. Wagner and Harvey [9] found parameter uncertainties to be lowest when DaI was on the order of 1 . When DaI values are much greater than 1, in some cases, most if not all solute undergoes some exchange into the storage zone and the storage zone parameters can only be estimated with large uncertainties. The experimental Damköhler numbers found from the transient storage modeling conducted here are listed in Table 1. Our DaI values were found to be within acceptable limits. 

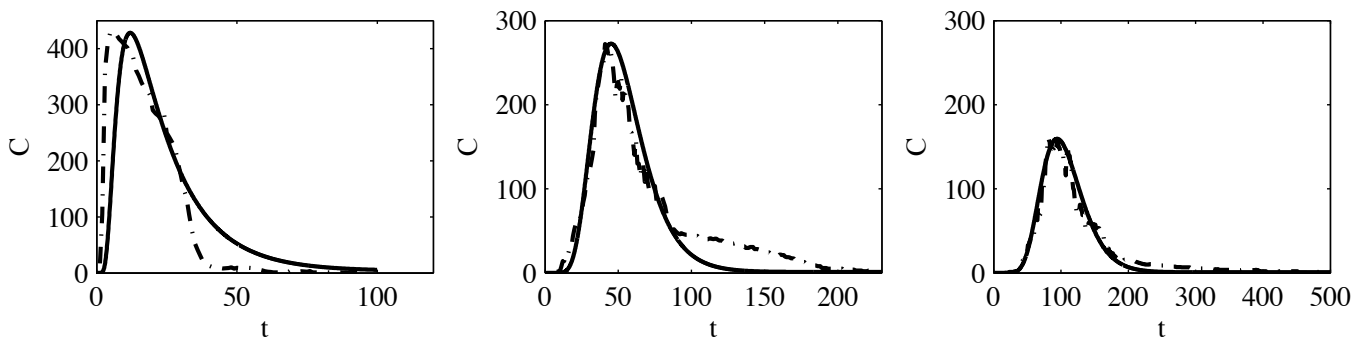

Figure 2: Solute concentration, C, in main stream. Numerical Solution (-); Experimental results $(-\cdot-)$ ): (a) I.P2; (b) I.P5; (c) I.P8.
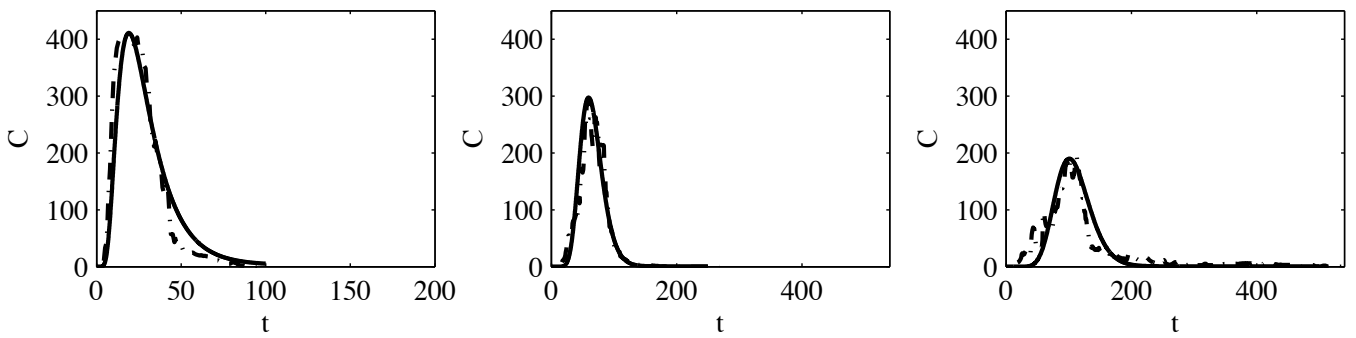

Figure 3: Solute concentration, C, in main stream. Numerical Solution (-); Experimental results $(-\cdot-$ ): (a) II.P2; (b) II.P5; (c) II.P8. 
Figures 2-3 show the results for the assays I.P2, I.P5 and I.P8 in clean conditions and for the assays II.P2, II.P5 and II.P8, after an operating period of eight months under organic loading conditions. The numerical solution seems to perform quite well for these cases.

The results show that the changes observed in the bed from Series I to Series II (the development of plants, roots and biofilm and the retention of solid material) did not seem to influence the hydrodynamic characteristics of the overall bed. A small amount of dispersion occurred (Pe between 40 and 60) and the flow regime was plug flow. However, a strong dispersion in the inlet section (Pe between 5 and 12) was observed. For clean conditions (I.P2), this circumstance may be explained by the occurrence of mixing and molecular diffusion (both longitudinal and axial) due to the closeness of the feeding point and the presence of a solute concentration gradient, respectively. The lower dispersion observed for the colonized bed (II.P2) seemed to be associated with the significant development of dead zones and the consequent occurrence of short-circuiting. The differences observed in D and Pe for clean and colonized conditions may be explained by spatial variations of the effective porosity after the bed colonization.

\section{References}

[1] J. M. Bahr and J. Rubin. Direct comparison of kinetic and local equilibrium formulations for solute transport affected by surface reactions. Water Resource Res. 23(3):438-452, 1987. doi:10.1029/WR023i003p00438 C349

[2] k: E. Bencala and R. A. Walters. Simulation of solute transport in a mountain pool-and-riffle stream: a transient storage model. Water Resour Res, 19(3):718-724, 1983. doi:10.1029/WR019i003p00718 C342 
[3] K. Dekker and J. G. Verwer. Stability of Runge-Kutta Methods for Nonlinear Stiff Differential Equations. CWI Monograph 2, Elsevier, Amsterdam, 1984. C345, C346

[4] S. H. Keefe, L. B. Barber, R. L. Runkel, J. N. Ryan, D. M. McKnight and R. D. Wass. Conservative and reactive solute transport in constructed wetlands. Water Resource Res., 40: W01201, 2004. doi:10.1029/2003WR002130 C340

[5] C. J. Martinez and W. R. Wise. Analysis of constructed treatment wetland hydraulics with the transient storage model OTIS. Ecological Engineering, 20(3):211, 2003. doi:10.1016/S0925-8574(03)00029-6 C340

[6] C. F. Nordin and B. M. Troutman. Longitudinal dispersion in rivers: the persistence of skewness in observed data. Water Resour Res, 16(1):123-128, 1980. doi:10.1029/WR016i001p00123 C342

[7] R. L. Runkel and S. C. Chapra. An efficient numerical solution of the transient storage equations for solute transport in small streams. Water Resour Res, 29(1):211-215, 1993. doi:10.1029/92WR02217 C342, C343

[8] J. G. Verwer and J. M. Sanz-Serna. Convergence of Method of Lines Approximations to Partial Differential Equations. Computing, 33(3-4):297-313, 1984. doi:10.1007/BF02242274 C344

[9] B. J. Wagner and J. W Harvey. Experimental design for estimating parameters of rate-limited mass transfer: analysis of stream tracer studies. Water Resource Res., 33(7): 1731-1741, 1997. doi:10.1029/97WR01067 C349

\section{Author addresses}

1. A. Araújo, CMUC, Department of Mathematics, University of Coimbra, 3001-454 Coimbra, Portugal. 
mailto:ecs@mat.uc.pt

2. E. Sousa, CMUC, Department of Mathematics, University of Coimbra, 3001-454 Coimbra, Portugal.

3. A. Albuquerque, C-MADE, Department of Civil Engineering and Architecture, University of Beira Interior, 6201-001 Covilha, Portugal. 